\title{
Molecular characterization and heterogeneity of circulating tumor cells in breast cancer
}

\author{
Anna Jakabova $^{1} \cdot$ Zuzana Bielcikova $^{2} \cdot$ Eliska Pospisilova $^{1} \cdot$ Rafal Matkowski $^{3,4} \cdot$ \\ Bartlomiej Szynglarewicz $^{3,4}$ - Urszula Staszek-Szewczyk ${ }^{3,4}$ - Milada Zemanova ${ }^{2}$. \\ Lubos Petruzelka $^{2}$ - Petra Eliasova ${ }^{1}$ - Katarina Kolostova ${ }^{1} \cdot$ Vladimir Bobek $^{1,5,6,7,8}$ (1)
}

Received: 8 July 2017/ Accepted: 7 August 2017/Published online: 16 August 2017

(C) The Author(s) 2017. This article is an open access publication

\begin{abstract}
Introduction This study analyzes peripheral blood samples from breast cancer (BC) patients. CTCs from peripheral blood were enriched by size-based separation and were then cultivated in vitro. The primary aim of this study was to demonstrate the antigen independent CTC separation method with high CTC recovery. Subsequently, CTCs enriched several times during the treatment were characterized molecularly.
\end{abstract}

Vladimir Bobek

vbobek@centrum.cz

1 Department of Laboratory Genetics, University Hospital Kralovske Vinohrady, Srobarova 50, 10034 Prague, Czech Republic

2 Department of Oncology, First Faculty of Medicine, Charles University and General University Hospital in Prague, Prague, Czech Republic

3 Division of Surgical Oncology and Department of Oncology, Wroclaw Medical University, Wybrzeże Ludwika Pasteura 1, 50-367 Wrocław, Poland

4 Breast Unit, Lower Silesian Cancer Center, Wroclaw, Plac Hirszfelda 12, 53-413, Wrocław, Poland

5 Department of Surgery, University Hospital Motol, V Uvalu 84, 15006 Prague, Czech Republic

6 Faculty of Medicine, Charles University, V Uvalu 84, 15006 Prague, Czech Republic

7 Department of Thoracic Surgery, Masaryk's Hospital, Krajska zdravotni a.s., Socialni pece 3316/12A, 40113 Usti Nad Labem, Czech Republic

8 Department of Histology and Embryology, Wroclaw Medical University, Wybrzeże Ludwika Pasteura 1, 50-367 Wrocław, Poland
Methods Patients with different stages of BC $(N=167)$ were included into the study. All patients were candidates for surgery, surgical diagnostics, or were undergoing chemotherapy. In parallel, 20 patients were monitored regularly and in addition to CTC presence, also CTC character was examined by qPCR, with special focus on HER2 and ESR status.

Results CTC positivity in the cohort was $76 \%$. There was no significant difference between the tested groups, but the highest CTC occurrence was identified in the group undergoing surgery and similarly in the group before the start of neoadjuvant treatment. On the other hand, the lowest CTC frequencies were observed in the menopausal patient group (56\%), ESR+ patient group (60\%), and DCIS group (44.4\%). It is worth noting that after completion of neoadjuvant therapy (NACT) CTCs were present in $77.7 \%$ of cases. On the other hand, patients under hormonal treatment were CTC positive only in $52 \%$ of cases.

Discussions Interestingly, HER2 and ESR status of CTCs differs from the status of primary tumor. In $50 \%$ of patients HER2 status on CTCs changed not only from HER2+ to HER2-, but also from HER2- to HER2+ (33\%). ESR status in CTCs changed only in one direction from ESR+ to ESR-.

Conclusions Data obtained from the present study suggest that $\mathrm{BC}$ is a heterogeneous disease but CTCs may be detected independently of the disease characteristics in $76 \%$ of patients at any time point during the course of the disease. This relatively high CTC occurrence in BC should be considered when planning the long-term patient monitoring.

Keywords CTCs - Circulating tumor cells - Breast cancer · Cultivation · In vitro $\cdot$ MetaCell $\cdot$ Gene expression 


\section{Introduction}

Enumeration of circulating tumor cells (CTCs) has showed a prognostic role in various stages of the breast cancer (BC). Hormone receptors (estrogen and progesterone) and HER2 status of primary BC tumor have been established during standard clinical biopsies and are of crucial importance in the choice of treatment. Real-time tumor monitoring through CTC enumeration could be an important indicator of individual cancer development [1].

CTCs as biomarkers can offer some valuable information about a patient's tumor, if detection, separation, and characterization are performed in a reliable manner. Although occurrence of CTCs in patients' peripheral blood is often very low, enrichment methods can be introduced for CTC separation before their characterization. They are usually based on surface protein expression, size, density, electric charges, or deformability of CTCs.

This study analyzes peripheral blood samples from patients with BC. CTCs from peripheral blood were enriched by size-based separation and then cultivated in vitro. The primary aim of this study was to demonstrate the antigen independent high sensitive separation method and a possibility of molecular characterization of CTCs enriched several times during the treatment.

\section{Materials and methods}

\section{Patients}

To date 167 patients with diagnosed $\mathrm{BC}$ have been enrolled in the study in accordance with the Declaration of Helsinki. All patients were candidates for surgery, surgical diagnostics, or with planned or applied chemotherapy. Based on their informed consent, clinical data were collected from all participating patients. Basic cytopathological data are reported in Table 1 . For each patient, approximately $2 \times 8 \mathrm{~mL}$ of venous blood was drawn from the antecubital veins and placed into S-Monovette tubes (Sarstedt AG \& Co., Numbrecht, Germany) containing $1.6 \mathrm{mg}$ EDTA/mL blood as an anticoagulant. The samples were processed at room temperature using an isolation procedure completed within $24 \mathrm{~h}$ after the blood draw.

\section{CTCs enrichment and culture}

The recently introduced size-based separation method for viable CTC enrichment process (MetaCell ${ }^{\circledR}$, MetaCell s.r. o., Ostrava, Czech Republic) [2-6] is based on the filtration of peripheral blood through a porous polycarbonate
Table 1 Basic cytopathological characteristics of patients

\begin{tabular}{|c|c|c|}
\hline & $N$ & $(\%)$ \\
\hline \multicolumn{3}{|l|}{ Stage } \\
\hline 0 & 3 & 2 \\
\hline IA & 45 & 30 \\
\hline IIA & 64 & 42.7 \\
\hline IIB & 20 & 13.3 \\
\hline IIIA & 13 & 8.7 \\
\hline IIIB & 1 & 0.67 \\
\hline IIIC & 4 & 2.67 \\
\hline \multicolumn{3}{|c|}{ Histopathological features } \\
\hline Benign & 2 & 1.7 \\
\hline DCIS & 9 & 7.6 \\
\hline LCIS & 1 & 0.85 \\
\hline IDC (NST) & 76 & 65.6 \\
\hline ILC & 14 & 11.86 \\
\hline Mixed & 16 & 13.6 \\
\hline \multicolumn{3}{|l|}{ Menopausal status } \\
\hline Premenopausal & 65 & 39.39 \\
\hline Menopausal & 18 & 10.9 \\
\hline Postmenopausal & 82 & 49.7 \\
\hline \multicolumn{3}{|l|}{ Tumor size } \\
\hline $\mathrm{T} 1$ & 63 & 61.1 \\
\hline $\mathrm{T} 2$ & 36 & 34.9 \\
\hline $\mathrm{T} 3$ & 4 & 3.8 \\
\hline \multicolumn{3}{|l|}{ Nodal involvement } \\
\hline N0 & 56 & 56.5 \\
\hline N1 & 37 & 37.3 \\
\hline $\mathrm{N} 2$ & 6 & 6 \\
\hline \multicolumn{3}{|l|}{ Grading } \\
\hline G1 & 7 & 11.8 \\
\hline G2 & 24 & 40.6 \\
\hline G3 & 28 & 47.4 \\
\hline \multicolumn{3}{|c|}{ HR and HER2 status } \\
\hline HR + HER2+ & 16 & 11.7 \\
\hline HR- HER2+ & 7 & 5.1 \\
\hline HR + HER2- & 91 & 66.4 \\
\hline HR- HER2- & 23 & 16.8 \\
\hline
\end{tabular}

membrane (with pores of $8 \mu \mathrm{m}$ diameter). The minimum and maximum volume of the filtered peripheral blood may be adjusted up to $50 \mathrm{~mL}$ of fluid. The standard $8 \mathrm{~mL}$ peripheral blood sample from patients suffering from BC was transferred into the filtration tube. Gradual transfer of the blood in several steps is preferred to prevent blood clotting on the membrane filter. The peripheral blood flow is supported by capillary action of the absorbent touching the membrane filter. The filtered CTCs were observed immediately after filtration on the membrane. The control and presence of filtered CTCs immediately after isolation eliminates false negative results. The membrane filter is 
kept in a plastic ring that is transferred into the 6-well cultivation plate, $4 \mathrm{~mL}$ RPMI media is added to the filter top and CTCs are cultured on the membrane in vitro under standard cell-culture conditions $\left(37{ }^{\circ} \mathrm{C}, 5 \%\right.$ atmospheric $\mathrm{CO}_{2}$ ) and observed by inverted microscope. The CTCs were grown in FBS-enriched RPMI medium (10\%) for a minimum of 14 days on the membrane. Alternatively, the enriched CTC fraction can be transferred from the membrane and cultured directly on any plastic surface or a microscopic slide, or the separation membrane may be translocated on a microscopic slide. Microscopic slide is preferred if immunohistochemistry/immunofluorescence analysis is planned. If an immediate CTC analysis is awaited, the CTC fraction is transferred in PBS $(1.5 \mathrm{~mL})$ to a cytospin slide. The slide is then dried for $24 \mathrm{~h}$ and analyzed by histochemistry (May-Grünwald staining) and/or by automated immunohistochemistry protocols (Ventana, Benchmark Ultra, Roche) using standard differential diagnostic antibodies in the pathological evaluation process.

\section{Cytomorphological analysis}

The stained fixed cells captured on the membrane were examined using light microscopy in two steps: (i) screening at $\times 20$ magnification to locate the cells; (ii) observation at $\times 40 / \times 60$ magnification for detailed cytomorphological analysis. Isolated cells and/or clusters of cells of interest (immunostained or not) were selected, digitized, and the images were then examined by an experienced researcher and/or pathologist. CTCs were defined as cells with the following characteristics: (i) with a nuclear size $\geq 10 \mu \mathrm{m}$ ); (ii) irregular nuclear contour; (iii) visible cytoplasm, cells size over $15 \mu \mathrm{m}$; (iv) prominent nucleoli; (v) high nuclearcytoplasmic ratio; (vi) proliferation; (vii) actively invading cells creating 2D or 3D cell groups.

\section{Gene expression analysis (GEA)}

The key purpose of GEA was to compare gene expression of tumor-associated markers in the CTC-enriched fractions to that in the whole blood (white blood cells). Gene expression analysis can be performed to confirm the origin of the captured cells on the separation membrane. Gene expression analysis (GEA) allows up to 20 tumor-associated markers in RNA from different cell fractions to be tested within a single quantitative polymerase chain reaction (qPCR) run. Differential diagnostics markers for qPCR test are chosen in accordance with the expected diagnosis.

RNA is isolated from the whole blood and CTC-enriched fraction on the membrane. The CTC-enriched fraction of cells grown on the separation membrane in vitro (the so-called "membrane fraction") was used for RNA isolation.
Finally, CTC-gene expression analysis allows identification of the relative amount of tumor-associated (TA) markers in the whole blood and in CTC-enriched fractions. If the tumor-associated genes are highly expressed in the CTC fraction, a subsequent analysis of chemoresistanceassociated (CA) genes is performed. Molecular analysis helps to identify which type of chemotherapeutic agents may be of use in tumor therapy and assigned as personalized cancer therapy based on CTC.

The cells captured on the membrane are lysed by RLTbuffer with beta-mercapto-ethanol (Qiagen). RNA is then isolated using the RNeasy Mini Kit (Qiagen). RNA from the whole blood is isolated with a modified procedure and the quality/concentration of RNA is measured by NanoDrop (ThermoScientific). As there are only up to a few hundred cells on the membrane, the median concentration of RNA is quite low $(5-10 \mathrm{ng} / \mu \mathrm{l})$. High Capacity cDNA Reverse Transcription Kit (Life Technologies) was used for cDNA production. Gene expression analysis was performed using Taqman chemistry with Taqman MGBprobes for all the tested genes (Life Technologies).

The following genes associated with tumorigenic character and therapeutic potential in breast cancer were chosen for the multimarker GEA panel: ACTIN, CD45, CD68, EPCAM, MUC1, KRT18, KRT19, ESR, PGR, MAMMAGLOBIN, HER2, CD24, CD44. Additionally, genes associated with chemoresistance were tested (MRP1-10, MDR1, ERCC1).

\section{Statistical analysis}

All analyses were performed using clinicopathological information transformed into variables 0 and 1 if applicable for tested characteristics. Chi squared test, $t$ tests, cluster analysis, and correlation analysis of qPCR data were outperformed using GeneX (MultiD, SE) and GraphPadPrism versus 5 (Graphpad, US). $P$ value of less than 0.05 was considered statistically significant.

\section{Results}

The main focus of the study was to detect CTCs shortage in $\mathrm{BC}$ patients by a new methodological approach which is based on size-dependent separation of CTCs and subsequent cytomorphological evaluation. Cytomorphological evaluation using vital fluorescence microscopy approach (Fig. 1) enables further use of the viable captured cells for RNA/DNA analysis.

Patients diagnosed with different stages of breast cancer (BC) $(N=167)$ were included into the study. The patients were divided based on clinicopathological criteria and CTC presence was tested. Summary of the collected CTC positivity data is presented in Table 2 . 

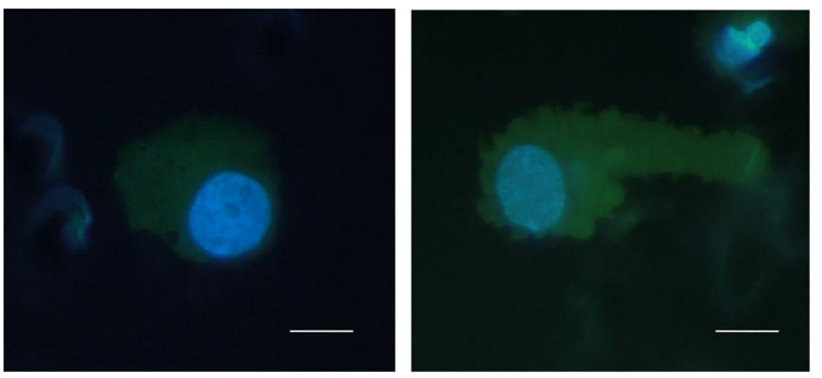

Fig. 1 CTCs isolated from a patient with breast cancer, captured on the separation membrane (vital fluorescent staining- NucBlue ${ }^{\circledR}$ and Celltracker $\left.{ }^{\circledR}\right)$. Bar represents $10 \mu \mathrm{m}$

CTC positivity in tested cohort was $76 \%$. There was no significant difference between tested subgroups, identifying a possible CTC presence, but the highest CTC occurrence was observed in the group undergoing surgery $(86.6 \%)$ and similarly in the group before the start of neoadjuvant and adjuvant treatment $(82.3 \%)$.

It is important to comment on relatively high CTCs presence even after neoadjuvant therapy has been completed $(77.7 \%)$. It can be assumed that in these patients therapy did not diminished all the cancer cell types.

There were no significant differences in CTC frequencies observed based on stage definitions. Considering the histopathological character of the primary tumor, the lowest CTC positivity was observed in DCIS (44.4\%). Relatively low CTC frequency was observed in the menopausal patient group (55.5\%).

Furthermore, it can be concluded that in tumors with ESR expression (ESR+) and without PGR expression (PGR-) CTCs were detected only in 60\% (9/15) of tested cases, whereas in ESR+/PGR + tumors CTC positivity was $73 \%$ (68/93). On the other hand, in patients with ESRnegative tumors CTCs were detected in $96.7 \%$ which is almost all of the patients under study (30/31). Therefore, it must be mentioned that during the therapy only $52.9 \%$ ( $9 /$ 17) of patients exhibited CTCs. Nevertheless, menopausal stage has to be considered if ESR/PGR expression is evaluated. The correlation of the menopausal status and ESR/PGR expression is illustrated in Fig. 2 which shows that hormonal receptor-positive tumors exhibit the lowest CTC detection frequencies in comparison to the HRgroups.

Similarly, even if not statistically significant, it can be seen that HR +/HER2 - tumors, irrespective of the menopausal stage show the lowest CTC frequency rates (see Fig. 3).

In parallel, 20 patients were monitored regularly during the course of the disease and in addition to CTC presence, CTCs character was also examined by qPCR with special focus on HER2 and ESR status. In total 43 qPCR analysis
Table 2 CTC positivity identified in BC-patient subgroups

\begin{tabular}{|c|c|c|}
\hline & $N$ & $(\%)$ \\
\hline CTC Positivity & $\mathrm{CTC}+$ & \\
\hline $\mathrm{CTC}+$ & 119 & 72.1 \\
\hline CTC- & 46 & 27.9 \\
\hline \multicolumn{3}{|l|}{ Stage } \\
\hline 0 & 3 & 100 \\
\hline IA & 31 & 68.9 \\
\hline IIA & 47 & 73.4 \\
\hline IIB & 16 & 80 \\
\hline IIIA & 10 & 76.9 \\
\hline IIIB & 0 & 0 \\
\hline IIIC & 4 & 100 \\
\hline \multicolumn{3}{|l|}{ Histopathological features } \\
\hline DCIS & 4 & 44.4 \\
\hline LCIS & 1 & 100 \\
\hline IDC (NST) & 55 & 72.4 \\
\hline ILC & 13 & 92.9 \\
\hline Mixed & 9 & 56.3 \\
\hline \multicolumn{3}{|l|}{ Menopausal status } \\
\hline Premenopausal & 51 & 78.4 \\
\hline Menopausal & 10 & 55.5 \\
\hline Postmenopausal & 58 & 70.7 \\
\hline \multicolumn{3}{|l|}{ Tumor size } \\
\hline $\mathrm{T} 1$ & 63 & 74.6 \\
\hline $\mathrm{T} 2$ & 36 & 88.8 \\
\hline $\mathrm{T} 3$ & 4 & 75 \\
\hline \multicolumn{3}{|l|}{ Nodal involvement } \\
\hline No & 23 & 82 \\
\hline N1 & 17 & 78 \\
\hline $\mathrm{N} 2$ & 2 & 66 \\
\hline \multicolumn{3}{|l|}{ Grading } \\
\hline G1 & 5 & 70 \\
\hline G2 & 14 & 58 \\
\hline G3 & 23 & 82 \\
\hline \multicolumn{3}{|l|}{ HR and HER2 status } \\
\hline $\mathrm{HR}+\mathrm{HER} 2+$ & 13 & 81.3 \\
\hline HR- HER2 + & 7 & 100 \\
\hline HR + HER2- & 64 & 70.3 \\
\hline HR- HER2- & 22 & 95.7 \\
\hline \multicolumn{3}{|l|}{ ESR + PGR + vs. ESR+ PGR- } \\
\hline \multicolumn{3}{|l|}{ Therapy } \\
\hline Before therapy & 28 & 82.3 \\
\hline During HT & 9 & 52.9 \\
\hline After NACT & 7 & 77.7 \\
\hline Before surgery (after biopsy) & 39 & 86.6 \\
\hline
\end{tabular}

were evaluated. Therapeutically, the most relevant findings are as follows: HER2 and ESR status of CTCs may differ from the status of primary tumor. 


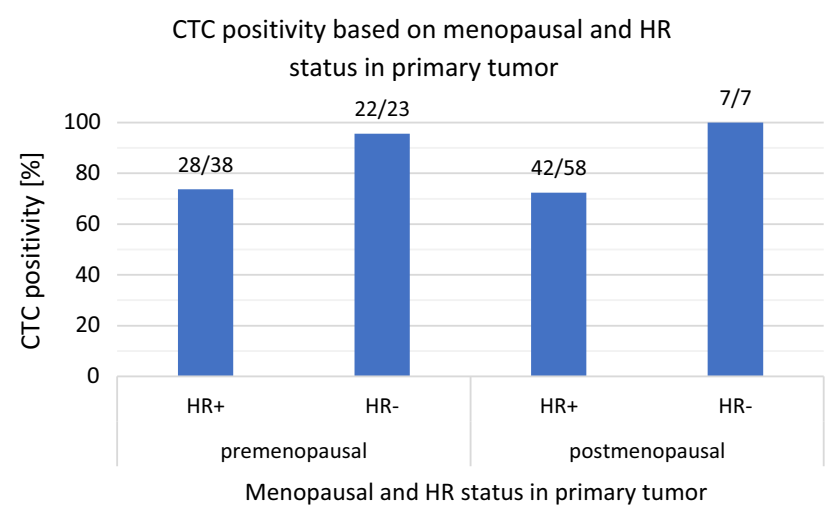

Fig. 2 CTC positivity in relation to menopausal stage and primary tumor $\mathrm{HR}$ - expression

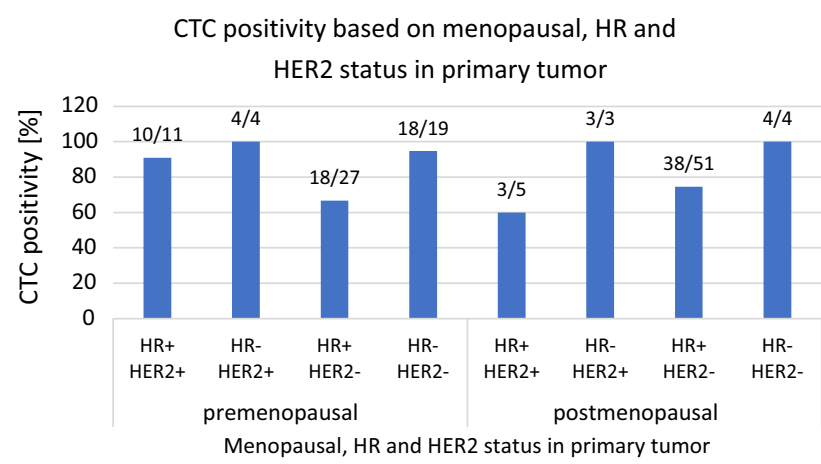

Fig. 3 CTC positivity in relation to menopausal stage and primary tumor HR and HER2- expression

The most frequent changes were seen in the triple negative BC (TNBC) group $(N=12)$ where 27 samples were evaluated. HER2 presence was confirmed in CTCs in four cases, which means that the change from HER2- to HER2+ occurred in $15 \%$ of tested samples, but that these four changes can be ascribed to four different patients. The change was relevant for four out of 12 patients (33.3\%) which is already a significant number. Similarly, in $50 \%$ of patients, HER 2 status changed from HER2 + to HER2- (3/6).

ESR status in CTCs changed only in one direction from ESR + to ESR- (3/3). These patients' primary tumors were diagnosed as ESR+/PGR+/HER2-. This group of patients will most probably exhibit very frequent changes.

Taken together, due to relatively high numbers of CTC positivity in different patient groups, we may conclude that a certain number of CTCs are always present in the blood of the patients. The cells have to be under selection pressure of treatment uninterruptedly. As soon as the selection pressure is stopped, new gene expression profile is displayed by CTCs.

The data obtained in the present study suggest that $\mathrm{BC}$ is a heterogeneous disease, but CTCs may be detected independently of the disease characteristics in 76\% (119/165) of patients at any time point of the course of the disease.
This relatively high CTC occurrence in BC should be considered in planning the long-term patient monitoring.

\section{Discussion}

Treatment decisions in $\mathrm{BC}$ are based on the characteristics of the primary tumor without considering the character of minimal residual disease or metastasis. However, tumors are evolving entities and genetic heterogeneity has been detected comparing the primary tumor with subsequent recurrences and metastases and analyzing different regions of the same tumor [7]. It has been hypothesized that the success of personalized treatments greatly depends on the capability to capture and monitor tumor heterogeneity over time and to consequently modulate therapies [8].

Detection and characterization of CTCs can contribute to the understanding of the disease and improved therapy monitoring as well as personalized treatment options. The key step is sensitive isolation and detection of CTCs. To date, various approaches have been also used to visually identify CTCs; however, the techniques employed to perform cell enrichment, immunohistochemical detection, and image analysis are complicated $[9,10]$. Moreover, epithelial markers are currently used to detect CTCs; tumor cells, however, may lose their epithelial features during metastasis/dissemination or may not express these markers because of their heterogeneity [11]. Therefore, some CTCs could be unidentified during epithelial-mesenchymal transition (EMT) by the common CTC-enrichment strategies relying on epithelial markers [12]. According to recent findings, more invasive CTCs may lose their epithelial antigens as a result of the EMT process [13] and EMT has been increasingly recognized as the key mechanism of cancer drug resistance [14].

We have used a simple method, without any complicated processing steps, for detecting viable human CTCs in the peripheral blood by using physical features of CTCs. We believe that viable CTCs may be a less invasive, repeatable biomarker for monitoring tumor responses.

In our study more than $76 \%$ patients were CTC positive. This result provides evidence that $\mathrm{BC}$ cells migrate and disseminate from morphologically very early lesions. Hosseini et al. demonstrated that metastatic dissemination often occurs early during tumor formation [15]. Disseminated cancer cells detected in patients before the manifestation of breast-cancer metastasis contain fewer genetic abnormalities than primary tumors and indicate that dissemination occurs during early stages of tumor growth [16-19].

As demonstrated by the SWOG S0500 trial, the simple enumeration of CTCs is not sufficient to guide therapy [20]. There is increasing evidence that cancer evolves over 
time because of its genomic instability and under the selection pressure of systemic treatments. These changes can be responsible for the appearance of drug-resistant clones. In studies of metastatic breast cancer (MBC), a discrepancy was observed between metastases or CTCs and the primary tumors in terms of HER2, estrogen and progesterone receptor expression [21, 22]. The loss of progesterone or estrogen hormone receptor expression in CTCs was described in $40 \%$ of receptor-positive MBC, while increased hormone receptor expression was detected in only $8 \%$ of triple negative MBC [21].

The clinical use of new CTC detection technique and the molecular characterization of isolated CTCs may lead to the development of personalized anticancer strategy in near future.

Funding The presented results of studies performed as part of the subject as per records in the Simple system number ST.C280.17.010 were financed through a statutory subsidy by the Polish Minister of Science and Higher Education.

\section{Compliance with ethical standards}

Conflict of interest The authors declare that they have no conflict of interest.

Open Access This article is distributed under the terms of the Creative Commons Attribution 4.0 International License (http://crea tivecommons.org/licenses/by/4.0/), which permits unrestricted use, distribution, and reproduction in any medium, provided you give appropriate credit to the original author(s) and the source, provide a link to the Creative Commons license, and indicate if changes were made.

\section{References}

1. Banys-Paluchowski M, Krawczyk N, Fehm T (2016) Potential role of circulating tumor cell detection and monitoring in breast cancer: a review of current evidence. Front Oncol 6:255

2. Kolostova K, Matkowski R, Jędryka M, Soter K, Cegan M, Pinkas M, Jakabova A, Pavlasek J, Spicka J, Bobek V (2015) The added value of circulating tumor cells examination in ovarian cancer staging. Am J Cancer Res 5:3363-3375

3. Kolostova K, Spicka J, Matkowski R, Bobek V (2015) Isolation, primary culture, morphological and molecular characterization of circulating tumor cells in gynecological cancers. Am J Transl Res 7:1203-1213

4. Kolostova K, Pinkas M, Jakabova A, Pospisilova E, Svobodova P, Spicka J, Cegan M, Matkowski R, Bobek V (2016) Molecular characterization of circulating tumor cells in ovarian cancer. Am J Cancer Res. 6(5):973-980

5. Kolostova K, Cegan M, Bobek V (2014) Circulating tumor cells in patients with urothelial tumors: enrichment and in vitro culture. CUAJ 8(9-10):E715
6. Kolostova K, Zhang Y, Hoffman RM, Bobek V (2014) In vitro culture and characterization of human lung cancer circulating tumor cells isolated by size exclusion from an orthotopic nudemouse model expressing red fluorescent protein. J Fluoresc 24 (5): $1531-1536$

7. Siu LL (2013) Accounting for tumor heterogeneity in the development of predictive biomarkers. Clin Adv Hematol Oncol $11: 312-313$

8. Gagan J, van Allen EM (2015) Next-generation sequencing to guide cancer therapy. Genome Med 7:80

9. Eliasova P, Kolostova K, Kobierzycki C, Bobek V (2013) Clinical studies monitoring circulating and disseminated tumor cells in gastrointestinal cancers. Folia Histochem Cytobiol 51(4):265-277

10. Liberko M, Kolostova K, Bobek V (2013) Essentials of circulating tumor cells for clinical research and practice. Crit Rev Hematol Oncol 88(2):338-356

11. Willipinski-Stapelfeldt B et al (2005) Changes in cytoskeletal protein composition indicative of an epithelial-mesenchymal transition in human micrometastatic and primary breast carcinoma cells. Clin Cancer Res 11:8006-8014

12. Mego M, De Giorgi U, Dawood S, Wang X, Valero V, Andreopoulou E, Handy B, Ueno NT, Reuben JM, Cristofanilli M (2011) Characterization of metastatic breast cancer patients with nondetectable circulating tumor cells. Int J Cancer 129:417423

13. Forte VA, Barrak DK, Elhodaky M, Tung L, Snow A, Lang JE (2016) The potential for liquid biopsies in the precision medical treatment of breast cancer. Cancer Biol. Med. 13:19-40

14. Du B, Shim JS (2016) Targeting epithelial-mesenchymal transition (emt) to overcome drug resistance in cancer. Molecules 21:7

15. Hosseini $\mathrm{H}$ et al (2016) Early dissemination seeds metastasis in breast cancer. Nature. doi:10.1038/nature20785

16. Schardt JA et al (2005) Genomic analysis of single cytokeratinpositive cells from bone marrow reveals early mutational events in breast cancer. Cancer Cell 8:227-239

17. Schmidt-Kittler O et al (2003) From latent disseminated cells to overt metastasis: genetic analysis of systemic breast cancer progression. Proc Natl Acad Sci USA 100:7737-7742

18. Hüsemann $Y$ et al (2008) Systemic spread is an early step in breast cancer. Cancer Cell 13:58-68

19. Sänger $N$ et al (2011) Disseminated tumor cells in the bone marrow of patients with ductal carcinoma in situ. Int $\mathrm{J}$ Cancer 129:2522-2526

20. Smerage JB, Barlow WE, Hortobagyi GN, Winer EP, LeylandJones B, Srkalovic G, Tejwani S, Schott AF, O'Rourke MA, Lew DL et al (2014) Circulating tumor cells and response to chemotherapy in metastatic breast cancer: SWOG S0500. J Clin Oncol 32:3483-3489

21. Aktas B, Kasimir-Bauer S, Muller V, Janni W, Fehm T, Wallwiener D, Pantel K, Tewes M (2016) DETECT Study Group. Comparison of the HER2, estrogen and progesterone receptor expression profile of primary tumor, metastases and circulating tumor cells in metastatic breast cancer patients. BMC Cancer 16:522

22. Hayes DF, Cristofanilli M, Budd GT, Ellis MJ, Stopeck A, Miller MC, Matera J, Allard WJ, Doyle GV, Terstappen LW (2006) Circulating tumor cells at each follow-up time point during therapy of metastatic breast cancer patients predict progressionfree and overall survival. Clin Cancer Res 12:4218-4224 